\title{
Measurement invariance of the Satisfaction with Life Scale by gender, age, marital status and educational level
}

\author{
Irene Checa ${ }^{1}$ Jaime Perales ${ }^{2} \cdot$ Begoña Espejo $^{1}$
}

Accepted: 22 November 2018

(c) Springer Nature Switzerland AG 2018

\begin{abstract}
Introduction The Satisfaction with Life Scale (SWLS) has shown strong evidence of acceptability, reliability, validity and invariance for gender, whereas there is mixed evidence of invariance by culture and age and the literature has not explored the roles of marital status and educational level. The SWLS should be invariant by marital status and educational level to be able to compare scores between groups. We aimed to explore the invariance of the SWLS by marital status and educational level. Method A convenience sample of 726 Spanish adults participated in a survey. We tested a one-factor model using confirmatory factor analysis. We tested the configural, metric and scalar invariance of the factorial structure of the SWLS by gender, age, marital status and level of education.

Results The results show a scalar invariance by gender and educational level and a partial scalar invariance by marital status. Women and individuals in a relationship show greater subjective well-being while no differences are observed among people with different educational levels.

Discussion The SLWS is valid for comparisons between genders, age, educational levels but not for marital status. It is essential to verify its invariance to interpret mean differences and significance values appropriately.
\end{abstract}

Keywords Invariance $\cdot$ Psychometrics $\cdot$ Gender $\cdot$ Age $\cdot$ Marital status $\cdot$ Educational level

\section{Introduction}

The interest in understanding and measuring subjective wellbeing (SWB) has grown exponentially in the last decades [1]. SWB can be divided into a cognitive (life satisfaction) and an affective component (positive and negative emotions or emotional balance). The cognitive component, life satisfaction, refers to the global cognitive judgement people make about their own lives according to personal criteria [2].

To our knowledge, the scale with the strongest psychometric properties to measure the cognitive component of SWB is the Satisfaction with Life Scale (SWLS) [3]. The SWLS has been adapted and validated in several languages [4]. All these adaptations have shown a one-dimensional structure, which has been confirmed in all studies assessing

Irene Checa

irene.checa@uv.es

Universitat de Valencia, Valencia, Spain

2 University of Kansas Alzheimer's Disease Center, Fairway, KS, USA its factor structure. However, a recent review showed that the SWLS is being used to compare groups that have not shown strict or scalar invariance [4].

Scales show measurement invariance (MI) when they measure the same construct in different subgroups. Therefore, $\mathrm{MI}$ is required before comparing means of such subgroups. There are four levels of invariance, configural, metric, scalar and strict [4]. The current literature overwhelmingly shows that scalar invariance is sufficient to assess comparisons between latent factor means [28, 29]. However, strict invariance is more appropriate for comparisons between observed factor means.

Some studies have also tested the invariance of the SWLS by gender [4]. Most studies have shown strict [5-9] or scalar [10-12] invariance, meaning that comparisons between men and women are appropriate. However, data have not fully supported invariance by age and culture as only one study among Russian and American students showed scalar invariance [13] and an Angolan study found strict invariance for six different age groups [9].

While most studies have focused their invariance testing on age, gender and culture, other studies have focused 
on differences in SWB measured with the SWLS by marital status $[14,15]$ but their data have not supported scalar invariance. However, studies using other measures of SWB have tested and showed invariance by marital status [27]. These studies showed that having a stable partner or being married predicts high levels of SWB possibly due to feelings of loneliness. However, no study has explored the invariance of the SWLS by marital status.

Educational level is also a good predictor of high levels of SWB [16] and there is a need to show stronger evidence of its role in the SWLS's invariance as only Bai et al. [5] observed metric invariance across Chinese adults of four educational levels. For this reason, the goal of this paper is to explore configural, metric and scalar invariance of the SWLS by marital status and educational level. We also report additional evidence of gender and age invariance in a large Spanish sample.

\section{Method}

\section{Participants}

A convenience sample of 726 Spanish adults 18-71 years, $(\mathrm{M}=29.36 ; \mathrm{SD}=12.37)$ participated in an online survey. Part of the sample included students of the Faculty of Psychology of the University of Valencia (52.2\%), who responded to the survey using their computers in the classroom. The rest of participants were recruited through mailing lists or social networks of the authors, as well as personal contacts via a survey link sent by email. All participants were asked about their nationality and this study only included those whose nationality was Spanish. To participate, all individuals had to read the first page of the online survey which summarized the project and emphasized their right to withhold participation in any part of the project and the anonymity of the data. Only individuals who clicked on the "agree to participate" option had access to the rest of the survey and were considered participants. Participation was voluntary, and participants had the option to delete their answers and leave the survey at all times. Demographic characteristics are shown in Table 1.

\section{Measures}

The Satisfaction with Life Scale (SWLS) is a five-item instrument designed to measure global cognitive judgment of satisfaction with one's own life [2,3]. Participants indicated how much they agreed or disagreed with each item using a seven-point Likert scale ranging from one (strongly disagree) to seven (strongly agree). Scores range from 7 to 35 , higher scores indicating higher life satisfaction.
Table 1 Sociodemographic characteristics of the sample

\begin{tabular}{lll}
\hline & $n$ & $\%$ \\
\hline Gender & 325 & \\
$\quad$ Male & 401 & 44.8 \\
Female & & 55.2 \\
Age & 375 & 51.7 \\
24 or younger & 351 & 48.3 \\
Older than 24 & & \\
Marital status & 458 & 64.5 \\
Single & 258 & 35.5 \\
In a relationship & \\
Educational level & & 55.0 \\
High school or lower & 399 & 45.0 \\
Higher than high school & 327 & \\
\hline
\end{tabular}

${ }^{\text {a }}$ Married, common-law and in a romantic relationship with another person

Regarding sociodemographic variables, educational level was obtained by asking about the highest level of education completed, with answers being primary studies (6-12 years), compulsory secondary studies (13-16 years), high school (non-compulsory, 17-18 years) and college studies or above. This variable was later categorized as high school or lower versus higher than high school. Marital status was categorized into single (never married, widowed, separated, divorced persons) versus in a relationship (married, common-law and in a romantic relationship with another person). Questions also asked whether participants were male or female and their age, which was later categorized as 24 years old or younger and older than 24 years old. The age groups were categorized with a cutoff of 24 given the high number of undergraduate students aged 24 and younger and the fact that splitting those 25-71 into subgroups was not feasible as the sample size would have been too small to test invariance.

\section{Data analysis}

We tested the one-factor structure widely supported in the literature [4] and conducted confirmatory factor analysis (CFA) using Mplus 8.1 [17]. We used maximum likelihood robust (MLR) to estimate model parameters and statistics for two reasons. First, most invariance studies using the SWLS treated data as continuous [4] and second, MLR is considered the best estimator with missing data and with Likert scales of more than five points [30]. We calculated Chi square $\left(\chi^{2}\right)$, comparative fit Index (CFI), Tucker-Lewis index (TLI) and root mean square error of approximation (RMSEA) to evaluate the goodness of fit of the proposed models. Cut-offs for goodness of fit were 0.90 for CFI/TLI 
and 0.08 for RMSEA indicating acceptable fit and 0.95 for CFI/TLI and 0.06 for RMSEA indicating excellent fit [18].

We assessed invariance of the factorial structure of the SWLS by gender, marital status and educational level. Three nested models with increasing degrees of restriction were tested: the base model assessed configural invariance and allowed free estimation of all the parameters for each group. The metric (weak) invariance model, nested in the configural model, added the restriction of invariant factor loadings among groups. Finally, the scalar (strong) invariance model, nested in the second model, added the intercept constraint of the invariant items among the comparison groups. Given that the Chi-square indices are sensitive to the sample size, we focused mainly on the comparison of the CFI, TLI and RMSEA indices. We considered a variation of these indices higher than .01 as a criterion to rule out the invariance of the more restrictive model and accept the more parsimonious model [19]. We also assessed composite reliability of the scale using Raykov's coefficient. We considered values to be acceptable if they reached 0.70 [20].

\section{Results}

Table 2 shows the descriptive data of the SWLS for the complete sample and for each of the groups in which the invariance was measured. Composite reliability (Raykov's coefficient) was good (.869). The one-dimensional model for the total sample presented an excellent fit $\chi^{2}=14.610(5 \mathrm{df})$, $\mathrm{CFI}=.991 ; \mathrm{TLI}=.982 ; \mathrm{RMSEA}=.052$.
Table 3 shows the goodness-of-fit indices of the multidimensional model by gender, age, marital status and educational level and nested models of invariance, in ascending order of restriction level. Results show that the SWLS had strong invariance by gender, and the fit of the one-dimensional model for male and female was excellent. These results mean that the latent means can be compared by gender. The latent mean values were fixed to zero for males and women had a greater satisfaction than men $(b=.152$, $z=2.028, p<.05$ ).

The model showed acceptable fit for both age groups, meaning that the latent means of both groups can be compared because the SWLS presented strong invariance by age. The latent mean values were fixed to zero for individuals 24 and younger. In this case, there were no significant differences in satisfaction with life by age $(b=-.002, z=-.024$, $p=.981)$.

The model showed acceptable fit for both marital status groups. However, in this case, invariance was not included in the most restrictive model (scalar). Partial scalar invariance was calculated by keeping item 2 invariant ("The conditions of my life are excellent"). Assuming this partial invariance, both groups can be compared by marital status and those in a relationship were more satisfied with their life than single individuals $(b=.191 ; z=2.486 ; p<.05)$, but item 2 has been fixed to 0 in both groups.

The model fitted excellently for participants with high school or lower educational level and acceptably for those with an educational level higher than high school, meaning that the latent means of both groups can be compared because the SWLS presented strong invariance by
Table 2 Items' means and standard deviations, for the total sample and all subgroups

\begin{tabular}{|c|c|c|c|c|c|c|c|c|c|c|}
\hline & \multicolumn{2}{|l|}{ I1 } & \multicolumn{2}{|l|}{$\mathrm{I} 2$} & \multicolumn{2}{|l|}{ I3 } & \multicolumn{2}{|l|}{ I4 } & \multicolumn{2}{|l|}{ I5 } \\
\hline & Mean & SD & Mean & SD & Mean & SD & Mean & SD & Mean & SD \\
\hline Total sample & 3.66 & 1.11 & 3.81 & 1.07 & 3.91 & 1.01 & 3.27 & 1.22 & 4.07 & .954 \\
\hline \multicolumn{11}{|l|}{ Gender } \\
\hline Male & 3.56 & 1.12 & 3.67 & 1.11 & 3.85 & 1.02 & 3.20 & 1.26 & 4.09 & .911 \\
\hline Female & 3.75 & 1.09 & 3.92 & 1.03 & 3.95 & 1.00 & 3.33 & 1.19 & 4.06 & .988 \\
\hline \multicolumn{11}{|l|}{ Age } \\
\hline 24 or younger & 3.65 & 1.14 & 3.77 & 1.22 & 3.90 & 1.26 & 3.37 & 1.50 & 4.06 & .982 \\
\hline Older than 24 & 3.67 & 1.27 & 3.85 & 1.07 & 3.91 & 1.00 & 3.16 & 1.47 & 4.08 & .831 \\
\hline \multicolumn{11}{|l|}{ Marital status } \\
\hline Single & 3.59 & 1.19 & 3.66 & 1.11 & 3.84 & 1.04 & 3.23 & 1.25 & 4.05 & .966 \\
\hline In a relationship & 3.80 & 1.07 & 4.09 & .940 & 4.03 & .927 & 3.35 & 1.18 & 4.11 & .933 \\
\hline \multicolumn{11}{|l|}{ Educational level } \\
\hline High school or lower & 3.65 & 1.09 & 3.71 & 1.10 & 3.91 & 1.01 & 3.26 & 1.21 & 4.02 & .987 \\
\hline Higher than high school & 3.68 & 1.12 & 3.93 & 1.03 & 3.90 & 1.00 & 3.28 & 1.24 & 4.13 & .910 \\
\hline
\end{tabular}

Item (1) In most ways my life is close to my ideal; Item (2) The conditions of my life are excellent; Item (3) I am satisfied with my life; Item (4) So far I have gotten the important things I want in life; Item (5) If I could live my life over, I would change almost nothing

SD Standard deviation 
Table 3 Tested models and goodness-of-fit indices

\begin{tabular}{|c|c|c|c|c|c|c|c|c|c|}
\hline & $\chi^{2}$ & $d f$ & $\Delta \chi^{2}$ & $\Delta d f$ & CFI & TLI RMSEA & $\Delta \mathrm{CFI}$ & $\Delta \mathrm{TLI}$ & $\Delta$ RMSEA \\
\hline \multicolumn{10}{|l|}{ Models in each group } \\
\hline \multicolumn{10}{|l|}{ Gender } \\
\hline Female & $10.939 *$ & 5 & & & .993 & .986 .055 & & & \\
\hline Male & $8.287 *$ & 5 & & & .995 & .990 .046 & & & \\
\hline \multicolumn{10}{|l|}{ Age } \\
\hline 24 or younger & $16.657 *$ & 5 & & & .979 & .958 .079 & & & \\
\hline Older than 24 & 2.037 & 5 & & & .00 & $1.01 \quad .000$ & & & \\
\hline \multicolumn{10}{|l|}{ Marital status (partial scalar) } \\
\hline Single & $19.008 *$ & 5 & & & .986 & .971 .078 & & & \\
\hline In a relationship & $11.612 *$ & 5 & & & .990 & .979 .074 & & & \\
\hline \multicolumn{10}{|l|}{ Educational level } \\
\hline High school or lower & $14.117 *$ & 5 & & & .999 & .998 .067 & & & \\
\hline Higher than high school & $13.695^{*}$ & 5 & & & .989 & .977 .075 & & & \\
\hline \multicolumn{10}{|c|}{ Global models } \\
\hline \multicolumn{10}{|l|}{ Gender } \\
\hline Configural & $19.226^{*}$ & 10 & - & - & .994 & .988 .051 & - & - & - \\
\hline Metric & $24.529 *$ & 14 & 5.302 & 4 & .993 & .990 .046 & -.001 & .002 & -.005 \\
\hline Scalar & $38.716^{*}$ & 18 & 14.187 & 4 & .987 & .985 .057 & -.006 & -.005 & .009 \\
\hline \multicolumn{10}{|l|}{ Age } \\
\hline Configural & $23.909 *$ & 10 & - & - & .992 & .983 .063 & - & - & - \\
\hline Metric & $26.742 *$ & 14 & 2.833 & 4 & .992 & .989 .051 & .000 & .006 & -.012 \\
\hline Scalar & $39.091 *$ & 18 & 12.350 & 4 & .987 & .986 .057 & -.005 & -.003 & .006 \\
\hline \multicolumn{10}{|l|}{ Marital status } \\
\hline Configural & $30.620^{*}$ & 10 & - & - & .987 & .974 .077 & - & - & - \\
\hline Metric & $34.304 *$ & 14 & 3.683 & 4 & .987 & .982 .064 & .000 & .008 & .013 \\
\hline Scalar & $58.117^{*}$ & 18 & 23.813 & 4 & .975 & 972.080 & -.012 & -.010 & -.016 \\
\hline Partial scalar & $36.164^{*}$ & 17 & 1.860 & 3 & .988 & .986 .057 & .001 & .004 & .007 \\
\hline \multicolumn{10}{|l|}{ Educational level } \\
\hline Configural & $28.076^{*}$ & 10 & - & - & .989 & .977 .072 & - & - & - \\
\hline Metric & $31.849 *$ & 14 & 3.772 & 4 & .989 & .984 .060 & .000 & .007 & -.012 \\
\hline Scalar & $47.429 *$ & 18 & 15.580 & 4 & .982 & 979.068 & -.007 & -.005 & .008 \\
\hline
\end{tabular}

$d f$ degrees of freedom, $\chi^{2}$ Chi square, $\Delta \chi^{2}$ difference in Chi square, $\Delta d f$ difference in degrees of freedom, $C F I$ comparative fit index, TLI Tucker-Lewis index, RMSEA root mean square error of approximation, $\triangle C F I$ difference in comparative fit index, $\triangle T L I$ difference in Tucker-Lewis index, $\triangle R M S E A$ difference in root mean square error of approximation

*All of $\chi^{2}$ are $\mathrm{p}<.001$

educational level. The latent mean values were fixed to zero for individuals with high school education or lower. In this case, there were no significant differences in satisfaction with life by educational level $(b=.053, z=.710, p=.478)$.

\section{Discussion}

Satisfaction with life is a cognitive component of subjective well-being and it has been measured frequently with the SWLS. For this reason, it is important to test this measure's invariance among people of different groups before pointing out differences between those groups. This study provides for the first time evidence of the SWLS invariance in a sample of Spanish adults, based on educational level and marital status.

In this sample, the SWLS showed scalar invariance across genders, which is in line with previous studies [10-12]. Women showed higher life satisfaction than men in our sample [4]. Even though in some studies [21-23] item 2 has not been invariant for both genders, our results suggest that interpretations and the meaning of the SWLS may generally be considered as equivalent across genders.

Regarding the two age groups in our sample, the SWLS presented strong invariance by age and there were no significant differences in satisfaction with life by age group. Our study is the first to show scalar invariance by two groups of age: 24 or younger and older than 24 . These two groups 
were used as a reference of life change in previous SWLS invariance studies [6, 9, 10, 24]. In a previous study [9], the youngest group (14-17 years old) showed higher levels of SWB but latent means remained almost unchanged from 25 to 65 years old, in line with our findings.

The two new variables considered in this study have been marital status and educational level. In this case, the SWLS shows strong invariance between people with higher than high school education and those with a lower educational level, presenting in this sample similar values of satisfaction with their lives, in line with results obtained in previous studies [15, 25].

Regarding marital status, the SWLS did not show strong invariance due to item 2 . This item has also led to a lack of gender invariance in other studies [4]. We recommend that this item is not taken into account when comparing wellbeing by marital status. The calculation of partial scalar invariance allows us to compare the means of both groups and the results show that individuals in a relationship have higher levels of life satisfaction, like previous studies suggest [26].

This study has some limitations. The sample is not representative of the Spanish population, since it used nonprobabilistic sampling. In addition, future studies should examine the invariance of other scales of well-being, such as the Flourishing Scale, and compare results. Future research is also needed to compare across finer groups of age, education or marital status beyond binary groupings. These comparisons will require a much larger sample size.

The SWLS is still one of the most widely used scales and it is essential to assess its invariance among different groups to interpret mean differences and significance values appropriately. Invariance verification by educational level and marital status shows that comparing latent means between these groups is appropriate, although item 2 should not be taken into account when comparing by marital status.

Funding Research reported in this publication was supported by the National Institute on Aging of the National Institutes of Health under Award Number P30AG035982. The content is solely the responsibility of the authors and does not necessarily represent the official views of the National Institutes of Health.

\section{Compliance with ethical standards}

Conflict of interest The authors of this manuscript certify that they have no affiliations with or involvement in any organization or entity with any financial interest or non-financial interest in the subject matter or materials discussed in this manuscript.

Ethical approval Study procedures were determined by the University of València data control technician (and current director of the Chair of Privacy and Digital Transformation Microsoft-UV) as constituting non-identifiable, minimum risk survey research, in a private document signed electronically dated March 20th, 2015. Because it was an anony- mous survey study, approval by the ethical committee is not necessary, according to Spanish legislation.

Informed consent Informed consent was obtained from all individual participants included in the study.

\section{References}

1. Cummins, R. A. (2013). Measuring happiness and subjective wellbeing. In S. A. David, I. Boniwell \& A. Conley Ayers (Eds.), Oxford handbook of happiness (pp. 185-200). Oxford: Oxford University Press.

2. Diener, E., \& Emmons, R. A. (1984). The independence of positive and negative affect. Journal of Personality and Social Psychology, 47, 1105-1117. https://doi.org/10.1037/0022-3514.47.5.1105.

3. Diener, E., Emmons, R. A., Larsen, R. J., \& Griffin, S. (1985). The Satisfaction with Life Scale. Journal of Personality Assessment, 49(1), 71-75. https://doi.org/10.1207/s15327752jpa4901_13.

4. Emerson, S. D., Guhn, M., \& Gadermann, A. M. (2017). Measurement invariance of the Satisfaction with Life Scale: Reviewing three decades of research. Quality of Life Research, 26, 22512264. https://doi.org/10.1007/s11136-017-1552-2.

5. Bai, X., Wu, C., Zheng, R., \& Ren, X. (2011). The psychometric evaluation of the Satisfaction with Life Scale using a nationally representative sample of China. Journal of Happiness Studies, 12(2), 183-197. https://doi.org/10.1007/s10902-010-9186-x.

6. Clench-Aas, J., Nes, R. B., Dalgard, O. S., \& Aarø, L. E. (2011). Dimensionality and measurement invariance in the Satisfaction with Life Scale in Norway. Quality of Life Research, 20(8), 11311307. https://doi.org/10.1007/s11136-011-9859-x.

7. Moksnes, U. K., Løhre, A., Byrne, D. G., \& Haugan, G. (2014). Satisfaction with life scale in adolescents: Evaluation of factor structure and gender invariance in a Norwegian sample. Social Indicators Research, 118(2), 657-671. https://doi.org/10.1007/ s11205-013-0451-3.

8. Shevlin, M., Brunsden, V., \& Miles, J. (1998). Satisfaction with life scale: Analysis of factorial invariance, mean structures and reliability. Personality and Individual Differences, 25(5), $911-$ 916. https://doi.org/10.1016/S0191-8869(98)00088-9.

9. Tomás, J. M., Gutiérrez, M., Sancho, P., \& Romero, I. (2015). Measurement invariance of the Satisfaction with Life Scale (SWLS) by gender and age in Angola. Personality and Individual Differences, 85, 182-186. https://doi.org/10.1016/j. paid.2015.05.008.

10. Hultell, D., \& Gustavsson, J. P. (2008). A psychometric evaluation of the Satisfaction with Life Scale in a Swedish nationwide sample of university students. Personality and Individual Differences, 44(5), 1070-1079. https://doi.org/10.1016/j.paid.2007.10.030.

11. Jovanovic, V. (2016). The validity of the Satisfaction with Life Scale in adolescents and a comparison with single-item life satisfaction measures: A preliminary study. Quality of Life Research, 25(12), 3173-3180. https://doi.org/10.1007/s11136-016-1331-5.

12. Zanon, C., Bardagi, M. P., Layous, K., \& Hutz, C. S. (2014). Validation of the satisfaction with life scale to Brazilians: Evidences of measurement noninvariance across Brazil and US. Social Indicators Research, 119(1), 443-453. https://doi.org/10.1007/s1120 5-013-0478-5.

13. Tucker, K. L., Ozer, D. J., Lyubomirsky, S., \& Boehm, J. K. (2006). Testing for measurement invariance in the satisfaction with life scale: A comparison of Russians and North Americans. Social Indicators Research, 78(2), 341-360. https://doi. org/10.1007/s11205-005-1037-5. 
14. Blanchflower, D. G., \& Oswald, A. J. (2004). Well-being over time in Britain and the USA. Journal of Public Economics, 88(7-8), 1359-1386. https://doi.org/10.1016/S0047-2727(02)00168-8.

15. Dolan, P., Peasgood, T., \& White, M. (2008). Do we really know what makes us happy? A review of the economic literature on the factors associated with subjective well-being. Journal of Economic Psychology, 29(1), 94-122. https://doi.org/10.1016/j. joep.2007.09.001.

16. Ferrer-i-Carbonell, A., \& Gowdy, J. M. (2007). Environmental degradation and happiness. Ecological Economics, 60(3), 509516. https://doi.org/10.1016/j.ecolecon.2005.12.005.

17. Muthén, L. K., \& Muthén, B. O. (1998-2017). Mplus user's guide (8th ed.). Los Angeles: Muthén \& Muthén.

18. Hu, L. T., \& Bentler, P. M. (1999). Cutoff criteria for fit indexes in covariance structure analysis: Conventional criteria versus new alternatives. Structural Equation Modeling, 6(1), 1-55. https://doi. org/10.1080/10705519909540118.

19. Cheung, G. W., \& Rensvold, R. B. (2002). Evaluating goodnessof-fit indexes for testing measurement invariance. Structural Equation Modeling, 9(2), 233-255. https://doi.org/10.1207/S1532 8007SEM0902_5.

20. Raykov, T. (1997). Estimation of composite reliability for congeneric measures. Applied Psychological Measurement, 21(2), 173-184. https://doi.org/10.1177/01466216970212006.

21. Sovet, L., Atitsogbe, K. A., Pari, P., Park, M. S. A., \& Villieux, A. (2016). Psychometric evaluation of the satisfaction with life scale in Togo: A three-step approach. European Review of Applied Psychology, 66(5), 243-250, https://doi.org/10.1016/j. erap.2016.06.002.

22. Atienza, F. L., Balaguer, I., \& García-Merita, M. a. L. (2003). Satisfaction with life scale: Analysis of factorial invariance across sexes. Personality and Individual Differences, 35(6), 1255-1260. https://doi.org/10.1016/S0191-8869(02)00332-X.

23. Jovanović, V., Joshanloo, M., Đunda, D., \& Bakhshi, A. (2016). Gender differences in the relationship between domain-specific and general life satisfaction: A study in Iran and Serbia. Applied Research in Quality of Life, 12(1), 185-204. https://doi. org/10.1007/s11482-016-9461-z.
24. Glaesmer, H., Grande, G., Braehler, E., \& Roth, M. (2011). The German version of the Satisfaction with Life Scale (SWLS). European Journal of Psychological Assessment, 27(2), 127-132. https ://doi.org/10.1027/1015-5759/a000058.

25. Jongbloed, J. (2018). Higher education for happiness? Investigating the impact of education on the hedonic and eudaimonic wellbeing of Europeans. European Educational Research Journal, 17(5), 733-754. https://doi.org/10.1177/1474904118770818.

26. Yue Cao, J. S., Krause, L. L., Saunders, \& Jillian, M. R., \& Clark (2015). Impact of marital status on 20-year subjective well-being trajectories. Topics in Spinal Cord Injury Rehabilitation, 21(3), 208-217. https://doi.org/10.1310/sci2103-208.

27. Moghnie, L., \& Kazarian, S. S. (2012). Subjective happiness of lebanese college youth in Lebanon: Factorial structure and invariance of the Arabic Subjective Happiness Scale. Social Indicator Research, 109(2), 203-210. https://doi.org/10.1007/s1120 5-011-9895-5.

28. Van de Schoot, R., Lugtig, P., \& Hox, J. (2012). A checklist for testing measurement invariance. European Journal of Developmental Psychology, 9(4), 486-492. https://doi.org/10.1080/17405 629.2012.686740.

29. Vandenberg, R. J., \& Lance, C. E. (2000). A review and synthesis of the measurement invariance literature: Suggestions, practices, and recommendations for organizational research. Organizational Research Methods, 3(1), 4-70. https://doi.org/10.1177/10944 2810031002.

30. Finney, S. J., \& DiStefano, C. (2006). Non-normal and categorical data in SEM. In R. Hancock \& R. O. Mueller (Eds.), Structural equation modelling: A second course (pp. 269-314). Greenwich: Information Age Publishing.

Publisher's Note Springer Nature remains neutral with regard to jurisdictional claims in published maps and institutional affiliations. 